\title{
Medienaufgaben der Kirche für die Zukunft
}

\author{
von Hans Maier
}

Hat die Kirche für die Kommunikation der Menschheit in der Zukunft einen Beitrag $\mathrm{zu}$ leisten? Diese Frage ist $\mathrm{zu}$ bejahen, denn dort, wo es um Menschen und Zukunft geht, ist Kirche gefordert, mitzuarbeiten, mitzudiskutieren, mitzuplanen, mitzuverantworten. Dies gilt auch für den publizistischen Bereich, den die katholische Kirche, wie Schmolke in seinem Buch „Die schlechte Presse - Katholiken und Publizistik zwischen ,Katholik' und ,Publik" " dargelegt hat, leider zu lange nicht sehr ernst genommen hat.

In der Gesellschaft von heute und morgen kann sich die Kirche den Fragen der Kommunikation, der Publizistik und des Journalismus nicht entziehen. Ihr fallen für die Zukunft vor allem drei Aufgaben zu:

a) Eine Intensivierung der Medienerziehung, um die Menschen vor einer Überflutung und Úberreizung durch die Massenmedien zu schützen.

b) Uber alle neu entwickelten Kommunikationsmöglichkeiten wie Kabelfernsehen, Bildtelefon und Bildschirmzeitung die personale und persönliche Kommunikationsfähigkeit des Menschen zu erhalten und zu fördern.

c) An der konkreten Gestaltung der Presse, des Hörfunks und Fernsehens mitzuwirken.

Die technischen Entwicklungen, die Fernsehen, Hörfunk und Presse erleben, werden vom Durchschnittsbïrger nicht registriert. Der Wechsel vom Blei- zum Lichtsatz wird nur vom geschulten Auge erkannt; die Konsequenzen, die sich für die Nachrichtenübermittlung und die Aktualität von Zeitungen durch diese technischen Verbesserungen ergeben, werden fast nur in Fachkreisen diskutiert. Ahnlich steht es mit technischen Neuschöpfungen im Bereich des Hörfunks und des Fernsehens, das durch das Kabelfernsehen eine neue Dimension bekommt. Kennzeichnend für alle Massenmedien ist der Gewinn an Aktualität. Der Rezipient wird immer näher an das unmittelbare Ereignis herangeführt. Er wird über den Bildschirm und den Äther Augen- und Ohrenzeuge von augenblicklichen Geschehnissen. Die Ereignisse werden direkt und universell erlebt. Die Information erfolgt total und so massiv, daß sie vom einzelnen kaum noch aufgenommen und verarbeitet sowie in ihrer Bedeutung und Konsequenz ausgelotet werden kann. Keine Ware ist heute verderblicher als eine Nachricht. Das wird im Jahr 2000 noch ausgeprägter sein.

\section{Weiterbildung für Rezipient und Kommunikator?}

Rezipient und Kommunikator sind in gleicher Weise von der Kommunationstechnik überfordert. Die Kommunikatoren, die Journalisten und Redakteure, werden immer mehr zu Operateuren, die mit modernsten technischen Mitteln Nachrichten redigieren

Prof. Dr. Hans Maier ist Staatsminister für Kultus und Bildung, München, und Präsident des Zentralkomitees der Deutschen Katholiken. 
und direkt in den Satz geben. Die Rezipienten werden zu einer wehrlosen Masse von Nachrichtenkonsumenten, die die Zusammenhänge zwischen Nachrichtenentstehung, Umsetzung und Verbreitung nicht mehr durchschauen. Es entsteht das Paradox, daß die Information in Desinformation umschlägt. Dadurch, daß die Medien umfassend, in alle Lebensbereiche hinein Menschen erreichen, informieren und beeinflussen können, sind sie zu einem Machtfaktor geworden. Sie werden daher immer stärker in die politischen Strategien der Parteien und Gewerkschaften einbezogen. Viele gesellschaftliche Gruppen versuchen, vor allem in Hörfunk und Fernsehen Einfluß zu gewinnen. Gegen die Tendenz, die Freiheit der öffentlich-rechtlichen Anstalten einzuschränken und parteipolitisch zu mißbrauchen, müssen sich besonders die Kirchen wenden, denn sie sind in besonderer Weise Wahrer der Freiheit. Die Kirchen tragen am wenigsten den Charakter von Interessenverbänden. Daher können sie in besonderer Weise für die Sicherung der Informationsfreiheit, die Wahrung der geistigen und sittlichen Fundamente unseres Lebens eintreten. Die Kirche wird in Zukunft mehr als bisher dafür einzutreten haben, daß die Medien nicht $\mathrm{zu}$ Instrumenten der Herrschenden werden. Eine Zukunftsaufgabe liegt darin, immer wieder Anstöße zu geben, darüber nachzudenken, ob die Kontrollinstanzen ausreichen und ob privatwirtschaftliche Initiativen im Hörfunk- und Fernsehbereich nicht eine wünschenswerte Bereicherung darstellen könnten. Es gilt, nicht nur Schwachstellen in vorhandenen Kommunikationssystemen aufzuzeigen, sondern auch Alternativen anzubieten.

So wie in der Zeitschriftenlandschaft eine Zunahme der Spezialliteratur zu beobachten ist, könnten sich künftig auch im Zuge der Breitbandkommunikation "Spezialsender" $^{*}$ herausbilden, die sich besonders mit Wirtschaft, Technik, Bildung, Politik, Sport, Theater etc. befassen. Die Informationsflut ist möglicherweise anders nicht mehr zu bewältigen. Wo wäre dann Kirche präsent? Nur in einem „Spezialkanal ${ }^{\star}$ Kirche?

\section{Medienpädagogik - Wegweiser für die Zukunft}

Mit der technischen Entwicklung der Medien hat die medienpolitische Aufklärung der Rezipienten nicht Schritt gehalten. Seit längerer Zeit gibt es zwar das Zauberwort "Medienpädagogik“. Hat sie jedoch positive Wirkungen erzielt? Durchschlagende Erfolge blieben der Medienpädagogik, in der man den eigentlichen wirksamen Schutz der Rezipienten vor einer Oberfremdung und Manipulation durch die Medien sehen muß, versagt. In der Schule fand die Medienerziehung bisher keinen festen Ort. Im Deutsch-, Religions- und Sozialkundeunterricht werden Medienfragen nur sporadisch aufgegriffen, aber nicht systematisch aufgearbeitet. In den Weiterbildungsprogrammen blieb die Medienerziehung bisher ebenfalls ein "Stiefkind“. Sie wird von den Bürgern nicht angenommen und von vielen Erwachsenenbildnern nicht voll als pädagogisches Ziel akzeptiert. Welche Unsicherheit in diesem Bereich herrscht, zeigt schon die verworrene Terminologie - Medienpädagogik, Medienerziehung, Medienkunde, Mediendidaktik.

Es ist auffallend, daß publizistische Tagungen in katholischen Akademien beispielsweise sehr schlecht besucht werden und häufig ausfallen müssen. Eine Analyse der Volkshochschulprogramme in Nordrhein-Westfalen von Klaus Detlef R. Breuer und Jürgen Hüther zeigt, daß sich nur bei etwa fünf Prozent der Volkshochschulen ein kontinuierliches Angebot zur Medienerziehung findet. Die kritische Auseinandersetzung mit den Medien ist offenbar nicht gefragt. Könnte es daran liegen, daß der 
durchschnittliche Konsument von Presse und Rundfunk sich seiner Stellung und Verantwortung im Kommunikationsgeflecht gar nicht bewußt werden möchte, da er dann seine Haltung und seine Gewohnheiten im Medienkonsum ändern müßte? Oder ist es Resignation gegenüber den mächtigen Rundfunkanstalten und Pressekonzernen? Oder sind gar die Vorurteile gegen Journalisten als "Schreiberlinge“, "Schreibtischtäter" und "Skandaljäger" so festgefahren?

Und doch ist gerade das Wissen über die Medien, die Kommunikationstechnik, die Kommunikationskanäle sowie die Medienpolitik der wirksamste Schutz gegen ein wehrloses Ausgeliefertsein. Die Medienerziehung sollte in der Weiterbildung, vor allem in der katholischen Weiterbildungsarbeit einen festen Platz erhalten. In ihr liegt der Schlüssel für eine vernünftige und verantwortete Bewältigung der Medienzukunft. Es gilt, den Menschen bewußt zu machen, daß die Beschäftigung mit der Medienpolitik notwendig ist und die technische Entwicklung der Medien brisante Konsequenzen enthält.

Eine gezielte Medienpädagogik eröffnete auch die Möglichkeit, den Wert der Informationsfreiheit und des Rechts auf freie Meinungsäußerung darzulegen. Die Verletzung dieser Menschenrechte wird leider in breiten Schichten der Bevölkerung nidht als sehr gravierend empfunden. Aus dem letzten Jahresbericht des Internationalen Presseinstituts (IPI) geht hervor, daß die Länder mit freier Presse immer noch eine verschwindende Minderheit unter den Staaten der Welt bilden. Die Einschränkung der Pressefreiheit und die Verfolgung von Journalisten hat weltweit zugenommen. Uber allem kommunikationstechnischem Fortschritt werden gerade die ethischen und moralischen Implikationen der Pressefreiheit, der Publizistik und der journalistischen Verantwortung vernachlässigt - Themenbereiche, wo die Kirche, ihre Autorität und Erfahrung gefordert sind. Werden diese Fragen in der Pastoral- oder Moraltheologie berücksichtigt? Müßten sie nicht künftig stärker beachtet werden?

Doch nicht nur die Konsumenten und Rezipienten bedürfen der Weiterbildung. Auch die Journalisten werden sidh, um für die Zukunft gerüstet zu sein, technisch und fachlich weiterbilden müssen. Wie in keinem anderen Beruf kommt es bei den Redakteuren darauf an, auf immer neue, immer mehr, immer schwierigere und immer rascher sich stellende Fragen ständig neue, zuverlässige Antworten zu finden. Dies gilt z. B. gerade für den Bereich der Politik in einer freiheitlichen Demokratie. Es genügt dabei nicht mehr, Information bloß bereitzustellen; dem Journalisten stellt sich vielmehr angesichts der geschilderten Entwicklung die nützliche Aufgabe, sie auch „verfügbar" zu machen: d. h., seinem „Zielpartner“ ist nur gedient, wenn durch journalistische Vermittlung und Bearbeitung Komplexität so reduziert wird, daß die Eigenart eines Problems und seine Zusammenhänge greifbar werden, daß sie auch der Universalität der Informationen in die begrenztere "Gegenwelt" (Otto Groth) des einzelnen übernommen werden können und seinem - politischen, sozialen, wirtschaftlichen - Wissen und Verhalten zur Verfügung stehen. Insofern ist Journalismus heute in der Tat Lebenshilfe für das Individuum, die Gesellschaft und den demokratischen Staat. Denn das Individuum kann künftig weder universelle Bildung noch den ganzen Umfang relevanten Wissens integrieren, dies kann nur das soziale Ganze der Gesellschaft. Wenn aber Individualität des einzelnen wie seine gesellschaftliche Integration verwirklicht, der Zerfall der Gesellschaft verhindert und die demokratische Qualität politischer Willensbildung im Staat gesichert werden sollen, müssen durch journalistische Vermittlung Information und Kommunikation über 
Tatsachen und Meinungen für den einzelnen wie für politische und soziale Gruppen im beschriebenen Sinn verfügbar sein.

Diese Funktion journalistischer Tätigkeit in der modernen Industriegesellschaft hat Max Weber 1919 in einer Bemerkung ausgedrückt, die vielleicht erst heute ihre volle Wahrheit entfaltet: Eine wirklich gute journalistische Leistung beanspruche mindestens ebensoviel Geist wie eine Gelehrtenleistung - nur sei die dem Journalisten zukommende Verantwortung größer.

\section{Persönliche Kommunikationsfähigkeit erbalten}

Über alle technischen Kommunikationsentwicklungen müssen wir in Zukunft darauf bedacht sein, die persönliche Kontakt- und Kommunikationsfähigkeit der Menschen zu erhalten. Es ist auffallend, daß gerade zu einem Zeitpunkt, wo die technischen Kommunikationsmittel ein Höchstmaß an Perfektion erreicht haben, Formen der Gruppendynamik, des Sensitivity- und Kommunikationstrainings in Ubung kommen, um die Kommunikationsfähigkeit in der persönlichen, unmittelbaren und ureigensten Form zu lernen und zu aktivieren. Konferenzschaltung, Bildtelefon und Bildschirmübertragungen können das persönliche Gespräch und die Versammlung von Menschen an einem gemeinsamen Ort nicht ersetzen. Es ist auffallend, daß trotz Fernsehen, Hörfunk, Presse und Telefon viele Menschen unter ihrer Einsamkeit leiden. Die Medien haben oftmals zu einer Verschärfung dieser Isolation beigetragen und nicht zum Abbau.

Daher ist es notwendig, daß wir uns im kirchlichen Raum vor einer Uberfremdung durch Kommunikationstechniken hüten. Alte Formen der Wallfahrt, bewährte Treffen wie Katholikentage oder Verbandstreffen werden durch die neuen Kommunikationstechniken nicht überflüssig, sondern notwendiger, weil auf ihnen eine Begegnung der Menschen stattfindet. Hier werden Nachrichten und Erfahrungen ausgetauscht, die nicht über den allgemeinen Nachrichtenkanal, über den Bildschirm oder den Ather laufen, hier findet auch ein Teil der nichtverbalen Kommunikation statt. Das gemeinsame Feiern ist eine wichtige Form der Kommunikation. Sie ist mehr und mehr verdrängt worden. Mittlerweile spüren wir die Verarmung, die hiermit eingetreten ist. Im Bereich Kirche, Theologie, Glaube gibt es Vorgänge und Aussagen, die sich nicht mit Kameras festhalten und über Atherwellen übertragen lassen. Der Glaube ist wesentlich an die Person und an die persönliche Erfahrung gebunden. Die Medien können zusätzliche Informationen über Kirche und Theologie liefern, sie können bestärken oder verunsichern. Professor Gerhard Schmidtchen hat in der Analyse "Zwischen Kirche und Gesellschaft" nachgewiesen, daß mit der Erhöhung der Kommunikationsmenge die Kritik an den Institutionen steige. Mehr und mehr werden institutionelle Selbstverständlichkeiten diskutierbar. Spannungen zwischen Ansprüchen des einzelnen in einem sich modernisierenden Gesellschaftssystem und den traditionellen Anforderungen der Institutionen bilden eine Quelle für Informationen von hohem publizistischen Nachrichtenwert. Der Kritik an Institutionen folgen psychisch notwendigerweise Distanzierungen, die einerseits die gesellschaftliche Reorganisationsfähigkeit erhöhen, andererseits die Orientierungsfähigkeit des einzelnen, seine psychische Sicherheit herabsetzen. Die entstabilisierende Wirkung der Medien erhöht nun wieder die Chance der Verhaltenssteuerung durch Nachrichtenströme. Den Wirkungen der Massenmedien muß gerade im kirchlichen Bereich die Überzeugung durch den einzelnen gegenübertreten. 


\section{Die Zukunft braucht Alternativen}

Kirche hat sich nicht nur theoretisch mit Fragen der Medien auscinanderzusetzen, sondern muß sie konkret mitgestalten. Sie darf nicht über ihnen stehen, sondern in ihnen. Die Kirchen sollten nur die fachlich qualifizierten Persönlichkeiten als Vertreter in die Gremien der öffentlich-rechtlichen Rundfunkanstalten entsenden, damit ein sachkundiges Mitarbeiten und Mitbestimmen in den Aufsichtsgremien möglich ist.

Mit „Publik" hatte die deutsche Kirche nach dem Konzil einen mutigen konkreten Schritt zur positiven Belebung der deutschen Presselandschaft getan. Dieser Versuch schlug leider aus vielerlei Gründen fehl. Zurück blieb in maßgeblichen Kreisen ein durchaus verständlicher "Publik"-Schock. Die Barrieren, die vor einem neuen publizistischen Experiment überwunden werden müssen, sind groß. Dennoch, der „Publik"-Schock muß überwunden werden, die Frage nach der konkreten Mitgestaltung der Christen in den Medien muß wieder gestellt und diskutiert werden dürfen. Sie ist auch für die gesamte Kirchenpresse von Interesse.

Seit Jahren fehlt z. B. eine christliche Jugendzeitschrift, die eine Alternative zum marktbeherrschenden "Bravo" darstellt. Will man die potentiellen Leser und Abonnenten des Jahres 2000 auch für das Abonnement einer kirchlichen Zeitung oder Zeitschrift gewinnen, dann muß man sie bereits in der Jugend mit Kirche und kirchlicher Publizistik bekannt machen. Die Jugend wird die medienpolitische Landschaft von morgen bestimmen.

\section{S U M M A R Y}

The Churches of the future will have three main areas of responsibility: They have to intensify media education in order to protect men from being overwhelmed by the media, and from being overstimulated by it. In spite of developments for a new communications media, the capacity for personal communication among men has to be protected and developed. A concrete professional co-operation of the Church in shaping the Press, Radio and TV will be needed.

\section{RÉSUME}

A l'avenir, ce seront surtout trois devoirs qui échoiront à l'Eglise: une intensification de l'éducation par les mass media afin de protéger l'homme contre une inondation et une surexcitation dûes aux mass media. Lors du développement de tout moyen de communication nouveau, la capacité de communication personnelle de l'homme doit être maintenue et encouragée. Une assistance qualifiée concrête à la réalisation de la presse, de la radio et de la télévision est absolument nécessaire.

\section{RES UMEN}

La Iglesia debe afrontar en el futuro tres cometidos: Intensificar la educación en relación con los medios de comunicación, para defender a los hombres de un exceso de informaciones y de una hiperexcitación producida por los medios de difusión. En el desarrollo de nuevos medios debe mantenerse y promoverse la capacidad de comunicación del hombre. Es necesaria una actuación concreta en la configuración de la prensa, radio y televisión. 